\title{
Compositional Trend in the Specks of Tantalite, Tourmaline and Beryl Hosted within Complex Basement Rocks Using Geophysical and Geochemical Methods of Exploration
}

\author{
B. S. Badmus, O. D. Akinyemi, *A. M. Gbadebo, P. Babatunde, O. T. Olurin \\ and S. A. Ganiyu \\ Department of Physics, Federal University of Agriculture, Abeokuta, Nigeria \\ *Department of Environmental Management and Toxicology, Federal University of Agriculture, Abeokuta, \\ Abeokuta, Nigeria
}

\begin{abstract}
Vertical Electrical Sounding (VES) using Schlumberger array was employed in data collection. Data acquisition was carried out along Oyan river bank with a total of twenty-three (23) soundings. This location was divided into two zones: VES $01-09$ and VES 10 -23. Rock samples hosting specks of tantalite, tourmaline and beryl were collected from the artisan pits within the study area and taken for geochemical analysis in the laboratory. The result of geochemical analyses revealed the quality of the solid minerals in terms of mineral compositions (major, trace and rare earth elements) while geophysical field measurement revealed the resistivity values, vertical and lateral distributions and depth of the solid minerals. The range of electrical resistivity values of these solid minerals as revealed by laboratory measurement is $19.4-31.1 \Omega \mathrm{m}$ while that of the host rock is $10.0-100.0 \Omega \mathrm{m}$ as obtained from field measurements. Specks of tantalite, tourmaline and beryl were suspected to be sparsely distributed in other VES locations except at VESO2 and 09, where vast deposits were suspected. Two litho-facies changes were observed and this revealed the economic values of these solid minerals. Black tourmalines, beryl and tantalite samples collected as specks within the study area were of low quality while pink tourmaline is of moderate quality on the basis of the chemical constituents contained.
\end{abstract}

Keywords: Specks, Litho-facies, Electrical Resistivity, Geochemical Analyses, Mineral Composition

\section{Introduction}

Geophysical and geochemical explorations were carried out to determine the compositional trends of specks of tantalite, tourmaline and beryl hosted within the basement rocks of Iwajowa area of Oyo State, southwestern Nigeria using two different approaches: Electrical resistivity method coupled with direct laboratory method using conductivity meter and laboratory analysis of rock samples collected from the site; to ascertain the quality of the samples in terms of mineral compositions.

The need to explore these earth treasures with modern day techniques and equipment arises when considering the factors like cost, time waste, risk and destruction of ecosystems as well as land form. These minerals are distributed unevenly across the globe, some are exogenic (seen at the surface of the earth) while some are endogenic (buried underneath the earth surface) [1]. The distribution of the mineralized pegmatite as being occurred in a broad belt stretching from about 400km from Ago-Iwoye area in the southwest to Bauchi area in the northeastern Nigeria by [2]. The identification of mineralized pegmatite in the Precambrian basement of southeastern Nigeria was carried out by [3]. Geophysical exploration techniques (magnetic and electrical resistivity) have been employed to map the oil sands deposits so as to obtain information on the subsurface conditions with a view on exploration activities [4]. Geoelectric mapping and characterization of limestone deposits of Ewekoro formation using Schlumberger arrangement was carried out to map both vertical and lateral extents of the deposit and establish the various litho-facies changes of Ewekoro limestone [5]. Geoelectric evaluation of mica schist deposit of area $\mathrm{J}_{4}$ south-western Nigeria was carried out using direct laboratory method to characterize the mica schist into different degrees of purity for both economic and commercial purposes [6]. Vertical electrical sounding (VES) has also been found applicable in determining the extent of inland of the gravel deposits being quarried at the bank of Otamiri River, a tributary of Imo River to estimate the reserve potential within a specified grid cell [7]. In 2006, The analysis of tourmaline samples from Liddicoatite Elbaite series from Anjanabonoina, Madagascar to determine the mineral compositions both in weight (wt.\%) and ppm was successfully carried out by [8] . In 1999, [9] carried out mineralogical and geochemical investigation of emerald and beryl mineralization of Pan-African belt of Egypt, in which two environments for localization of beryl mineralization were given as emerald schist and beryl specialized granitoid. 


\subsection{Location of the Study Area}

The study area lies within Oyo State which is bounded in the west by Benin Republic, in the south by Ogun State, in the north by Kwara and Osun State and in the east by Osun/Ondo State. It occupies a total area of $5 \mathrm{~km}^{2}$ of Iwajowa area with longitude between $\mathrm{N} 07^{\circ} 58^{\prime} 17^{\prime \prime}$ and $\mathrm{N} 07^{\circ} 58^{\prime} 26^{\prime \prime}$ and latitude between $\mathrm{E} 003^{\circ} 2^{\prime} 24^{\prime \prime}$ and $\mathrm{E} 003^{0} 2^{\prime} 28^{\prime \prime}$.

\subsection{Geology of the study area and pegmatite distribution within Nigeria}

The basement complex of southwestern Nigeria lies to the rest of the West African craton in the region of late Precambrian to early palaeozoic orogenesis. The basement complex rocks of Nigeria are composed predominantly of migmatitic and granitic gneisses; quartzites, slightly migmatised to unmigmatised metasedimentary schist and meta-igneous rock, charnockitic, gabbroic, and dioritic rock; and the members of the older granite suit mainly granites, granodiorites and syenites, [10].

Pegmatites are widely distributed within Nigerian basement complex rocks with a marked concentration of the mineralized pegmatites occurring in a broad belt extending for about 400km from AgoIwoye area in the southwest to Bauchi area in the northeast Nigeria ([3], [11], [12], and [13]). However, ([2], [14]) have also identified mineralized pegmatites in the Precambrian basement of southeastern Nigeria. This Pan-African belt continues southwest into northeast Brazil where analogous mineralized metal pegmatites also occur ([15], [16]). The pegmatites range in dimensions from a few metres to a kilometer in length while the width varies from a few centimeters to ten meters and more. Dykes with strike lengths between 300-700 meters are quite common. Although the majority of the pegmatites occur in the form of regular, tabular dykes with fairly constant dips and strikes, many of richly mineralized pegmatites occur as silt-like bodies with pronounced pinch and swell structures.

\subsection{Brief Description of Tourmaline, Tantalite and Beryl}

Tourmaline is a complex borosilicate mineral with varying amount of aluminum, iron, magnesium, sodium, lithium, potassium and sometimes other elements. It exists in different facies: red, pink, blue, green, brown, yellow, violet-red and black. Its general formula can be written as $\mathrm{XY}_{3} \mathrm{Al}_{6}(\mathrm{OH})_{4}\left(\mathrm{BO}_{3}\right)_{3}\left(\mathrm{Si}_{6} \mathrm{O}_{18}\right)$ where $\mathrm{X}=\mathrm{Na}, \mathrm{Ca}$ and $\mathrm{Y}=\mathrm{Al}, \mathrm{Fe}^{+3}, \mathrm{Li}, \mathrm{Mg}, \mathrm{Mn}^{+2}$. Tourmaline is found in two main geological formations: Igneous rocks (granite pegmatite) and in metamorphic rocks such as schist and marble, [17]. Tourmaline can also be found in minor amounts as grains sandstone. It is used as gemstone and in electronic instrumentation because of its piezo-electric and pyro-electric properties.

Tantalite is a natural mineral which exist as columbite-tantalite (COLTAN), since pure tantalite is very rare. It is chiefly found in granite pegmatite and as a detrital mineral in some places in considerable amounts. The chemical formula is $(\mathrm{Fe}, \mathrm{Mn})(\mathrm{Ta}, \mathrm{Nb})_{2} \mathrm{O}_{6}$. Tantalum oxide is used in camera lenses, $\mathrm{x}$-ray films and ink-jet printer. Tantalum powder is used in electronic instrumentations while tantalum fabricated sheets, plates, rods and wires are used in jet engines.

Beryl is a major ore of beryllium, it is found in the same geological formation as tourmaline. Its chemical formula is $\mathrm{Be}_{3} \mathrm{Al}_{2} \mathrm{Si}_{6} \mathrm{O}_{18}$ but has variable amount of $\mathrm{Na}, \mathrm{Li}, \mathrm{Ce}, \mathrm{Fe}, \mathrm{V}$ and $\mathrm{Cr}$. It exists in different facies: green, blue, red, yellow, colourless and pink. The amount of $\mathrm{V}$ and $\mathrm{Cr}$ determines the colour of beryl. It is used as gemstone and it is the major source of beryllium which is used in metallurgical companies for strengthening other metals. 
Figure 1 Geological Map of the Study Area

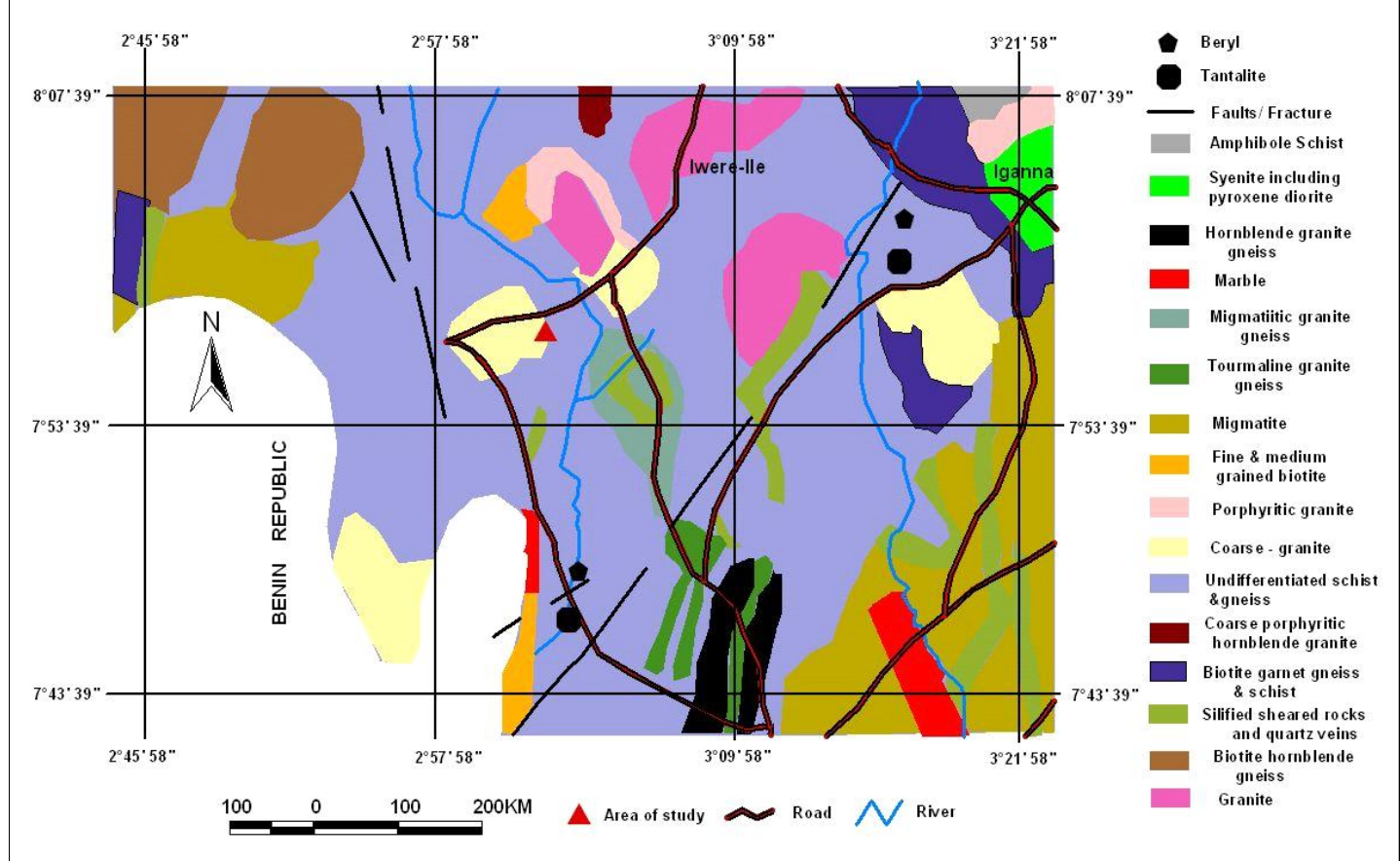

Figure1:Geological map of the study area

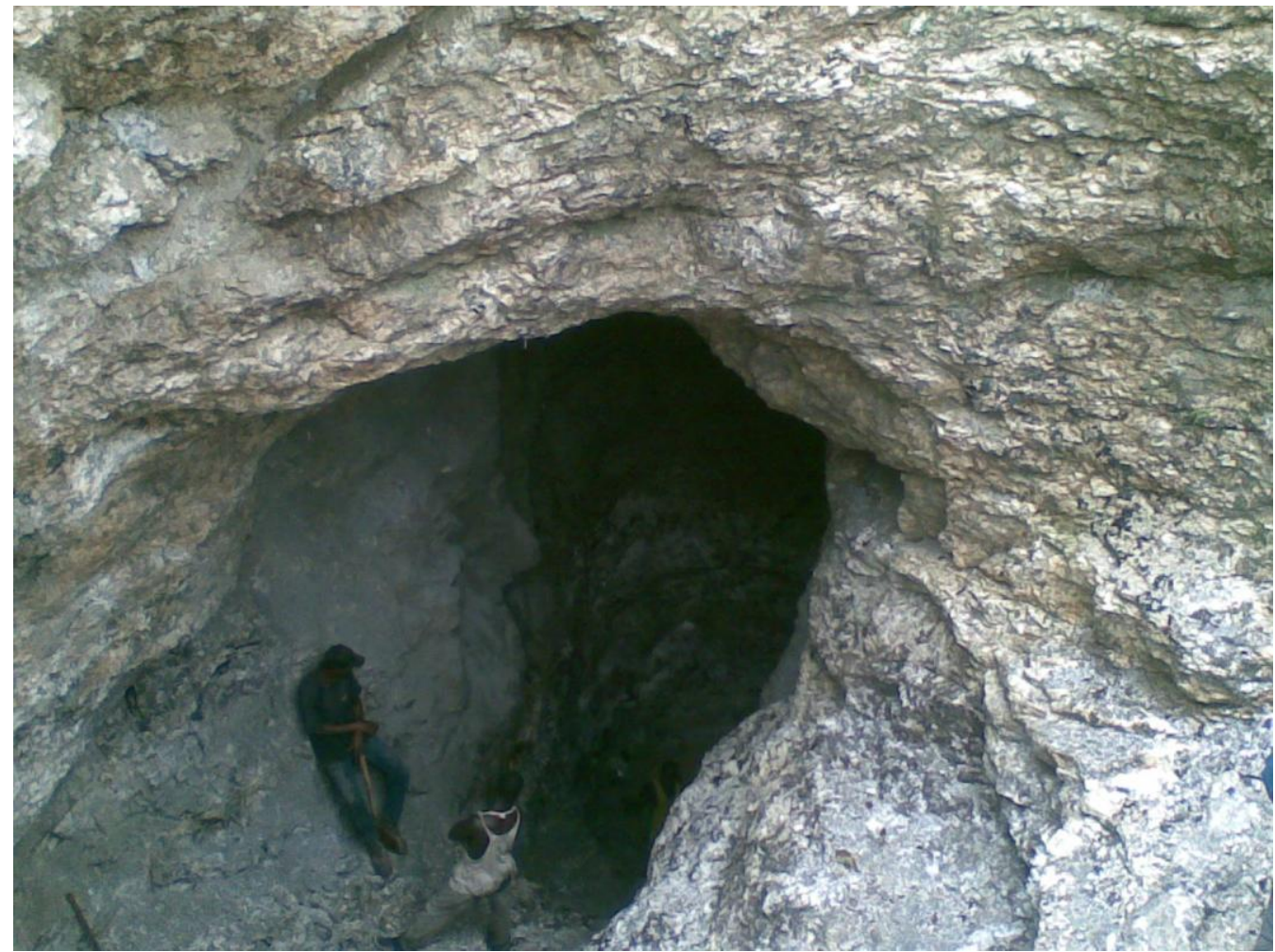

Figure 2: Pegmatite pit in which Artisan miners are carrying out mining activities.

\section{METHOD}

\subsection{Electrical Resistivity}

Electrical resistivity method using Schlumberger vertical electrical sounding was employed in data collection. Data acquisition was carried out along Oyan river bank with a total of twenty-three (23) soundings. Schlumberger array was used because of its greater depth of penetration [18]. These locations were divided into 
two zones: VES 01 - 09 and VES 10 - 23. The instruments used in carrying out the resistivity sounding include ABEM 300 SAS terrameter and its accessories. Rock samples labeled A, B, C, D, E, F, Q 1 and $\mathrm{Q}_{2}$ were collected from the Artisan pits within the study area and were taken for laboratory measurements. Electrical and thermal conductivities measurements of samples A (beryl), C (black tourmaline), F (tantalite) and Q (pink tourmaline) were carried out with the aid of conductivity meter. Thermal conductivity measurement was carried out on samples A, B, C, D, E and F. The preliminary interpretation was done using conventional curve matching method ([19], [20], and [21]). Based on the interpretation, initial estimates of resistivities and thickness of the various layers were obtained. These were used as initial model parameter for computer assisted interpretation technique for further refinement of the results used in final qualitative interpretation. RES 1D software was used for computer iteration technique. The results from computer modeling for the sounding stations VES (01-09) and VES (10-23) are summarized in Figure 5.

\subsection{Geochemical Analyses}

Geochemical analyses of rock samples collected from the site were carried out to determine their mineral compositions (in terms of major, trace and rare earth elements). All these analyses were carried out using Atomic Absorption Spectrophotometer (AAS) and their results are shown in Tables 5a, b, c and d.

\section{Result And Discussion}

TABLE 1: RESISTIVITY VALUES, DEPTH AND THE LITHOLOGY OF STUDY AREA (VES 01-09)

\begin{tabular}{|c|c|c|c|c|c|}
\hline VES LOCATION & $\begin{array}{l}\text { LAYE } \\
\text { R NO }\end{array}$ & $\begin{array}{l}\text { RESISTIVI } \\
\text { TY }(\Omega \mathrm{m})\end{array}$ & $\begin{array}{l}\text { THICKN } \\
\operatorname{ESS}(\mathrm{m})\end{array}$ & $\begin{array}{l}\text { DEPT } \\
\text { H (m) }\end{array}$ & LITHOLOGY \\
\hline \multirow{4}{*}{$\begin{array}{l}\text { VES 01 ELV:162m } \\
\text { :N 07 } 07^{0} 58^{\prime} 19^{\prime \prime} \\
\text { :E003 } 2^{\prime} 24^{\prime},\end{array}$} & 1 & 226.33 & 0.8 & 0.8 & Topsoil \\
\hline & 2 & 35.3 & 3.2 & 4.0 & Pegmatite \\
\hline & 3 & 1212.4 & 13.8 & 17.8 & Sandstone \\
\hline & 4 & 8308 & - & - & Fresh Basement \\
\hline \multirow{5}{*}{$\begin{array}{l}\text { VES } 02 \text { ELV:193m } \\
\text { Long:N07 } 58^{\prime} 18^{\prime \prime} \\
\text { Lat:E003 }{ }^{\circ} 26^{\prime},\end{array}$} & 1 & 2989.8 & 0.6 & 0.6 & Topsoil \\
\hline & 2 & 380.4 & 2.0 & 2.6 & Sandy Clay \\
\hline & 3 & 1946.8 & 3.1 & 5.7 & Clayey Sand \\
\hline & 4 & 68.2 & 24.2 & 30 & Pegmatite+Tourmaline \\
\hline & 5 & 2632.8 & - & - & Fresh Basement \\
\hline \multirow{4}{*}{$\begin{array}{l}\text { VES } \\
\text { ELV:228m } \\
\text { Long:N } 07^{0} 58^{\prime}, 20^{\prime \prime} \\
\text { Lat:E003 } 2^{\circ} 28^{\prime},\end{array}$} & 1 & 1770.9 & 0.4 & 0.4 & Topsoil \\
\hline & 2 & 91.4 & 1.2 & 1.6 & Weathered Granite \\
\hline & 3 & 394.2 & 29.3 & 30.9 & Clayey Sand \\
\hline & 4 & 10.6 & - & - & Tourmaline Zone \\
\hline \multirow{5}{*}{$\begin{array}{l}\text { VES } \\
\text { ELV:225m } \\
\text { Long:N07 }{ }^{0} 58^{\prime}, 20^{\prime}, \\
\text { Lat:E003 } 2^{\prime} 27^{\prime},\end{array}$} & 1 & 884.9 & 0.7 & 0.7 & Topsoil \\
\hline & 2 & 39.7 & 6.6 & 7.3 & $\begin{array}{l}\text { Pegmatite } \\
\text { Tourmaline+ Tantalite }\end{array}$ \\
\hline & 3 & 550.2 & 6.3 & 13.6 & Sandstone \\
\hline & 4 & 261.4 & 4.3 & 17.9 & Clayey Sand \\
\hline & 5 & 5613.9 & - & - & Fresh Basement \\
\hline \multirow{5}{*}{$\begin{array}{l}\text { VES } 05 \text { ELV186m } \\
\text { Long:N07 } 58^{\prime} 20^{\prime \prime} \\
\text { Long:E003 } 2^{\prime} 27^{\prime \prime}\end{array}$} & 1 & 4486.9 & 0.4 & 0.4 & Topsoil \\
\hline & 2 & 2001.9 & 0.7 & 1.1 & Compacted Sandstone \\
\hline & 3 & 60522 & 4.3 & 5.4 & Fresh Basement \\
\hline & 4 & 13835.9 & 1.1 & 6.5 & Fresh Basement \\
\hline & 5 & 745.8 & - & - & Fractured Basement \\
\hline \multirow{4}{*}{$\begin{array}{l}\text { VES } 06 \text { ELV:185m } \\
\text { Long:N07 } 58^{\prime} 20^{\prime \prime} \\
\text { Lat:E003 } 2^{\prime} 27^{\prime},\end{array}$} & 1 & 1386.9 & 0.7 & 0.7 & Topsoil \\
\hline & 2 & 961.7 & 0.5 & 1.2 & Compacted Sandstone \\
\hline & 3 & 1811.2 & 1.5 & 2.7 & Fresh Basement \\
\hline & 4 & 3553.9 & - & - & Fresh Basement \\
\hline \multirow{4}{*}{$\begin{array}{l}\text { VES } 07 \text { ELV:183m } \\
\text { Long:N } 07^{0} 58^{\prime}, 19^{\prime}, \\
\text { Lat:E003 } 2^{\prime} 27^{\prime},\end{array}$} & 1 & 356.5 & 0.4 & 0.4 & Topsoil \\
\hline & 2 & 18130.2 & 1.0 & 1.4 & Fresh Basement \\
\hline & 3 & 100000 & 29.1 & 30.5 & Fresh Basement \\
\hline & 4 & 72305.5 & - & - & Fresh Basement \\
\hline \multirow{4}{*}{$\begin{array}{l}\text { VES } 08 \text { ELV:209m } \\
\text { Long:N } 07^{0} 58^{\prime} 21^{\prime}, \\
\text { Lat:E003 } 2^{\prime} 28^{\prime},\end{array}$} & 1 & 118.2 & 1.1 & 1.1 & Topsoil \\
\hline & 2 & 36.5 & 3.8 & 4.9 & $\begin{array}{l}\text { Pegmatite (Tantalite+ } \\
\text { Tourmaline }\end{array}$ \\
\hline & 3 & 281.3 & 6.5 & 11.4 & Clayey Sand \\
\hline & 4 & 5832.6 & - & - & Fresh Basement \\
\hline VES & 1 & 880.6 & 0.7 & 0.7 & Topsoil \\
\hline
\end{tabular}


Compositional Trend in the Specks of Tantalite, Tourmaline and Beryl Hosted within Complex

\begin{tabular}{|l|l|l|l|l|l|}
\hline $\begin{array}{l}\text { ELV:212m } \\
\text { Long:N 07 } 58^{\prime}, 20 \\
\text { Lat:E003 }{ }^{0} 2^{\prime} 8^{\prime},\end{array}$ & 2 & 32.8 & 1.7 & 2.3 & $\begin{array}{l}\text { Pegmatite (Tourmaline } \\
+ \text { Associated Solid } \\
\text { Mineral) }\end{array}$ \\
\cline { 2 - 6 } & 3 & 1074.5 & 5.3 & 7.6 & Compacted Sandstone \\
\cline { 2 - 6 } & 4 & 77.5 & 27.5 & 35.2 & $\begin{array}{l}\text { Pegmatite } \\
\text { (Tourmaline+Tantalite }\end{array}$ \\
\cline { 2 - 6 } & 5 & 8300.9 & - & - & Fresh Basement \\
\hline
\end{tabular}

TABLE 2: RESISTIVITY VALUES, DEPTH AND LITHOLOGY OF THE STUDY AREA (VES 10 - 23)

\begin{tabular}{|c|c|c|c|c|c|}
\hline $\begin{array}{l}\text { VES } \\
\text { LOCATION }\end{array}$ & $\begin{array}{l}\text { LAYER } \\
\text { NO }\end{array}$ & $\begin{array}{l}\text { RESISTIVITY } \\
(\Omega \mathrm{m})\end{array}$ & $\begin{array}{l}\text { THICKNESS } \\
(\mathrm{m})\end{array}$ & $\begin{array}{l}\begin{array}{l}\text { DEPTH } \\
(\mathrm{m})\end{array} \\
\end{array}$ & LITHOLOGY \\
\hline \multirow{6}{*}{$\begin{array}{l}\text { VES } \\
\text { ELV:124m } \\
07^{0} 58^{\prime} 20^{\prime}, \\
\text { E:003' }\end{array}$} & 1 & 1913.4 & 0.4 & 0.4 & Topsoil \\
\hline & 2 & 92.8 & 0.7 & 1.0 & Pegmatite \\
\hline & 3 & 100.1 & 0.9 & 1.9 & Sandy Clay \\
\hline & 4 & 14016.2 & 8.8 & 10.7 & Fresh Basement \\
\hline & 5 & 4887.2 & 11.1 & 21.9 & Fresh Basement \\
\hline & 6 & 1384.2 & - & - & Fresh Basement \\
\hline \multirow{5}{*}{$\begin{array}{ll}\text { VES } & 11 \\
\text { ELV:155m } & \mathrm{N}: \\
07^{0} 58^{\prime} 19^{\prime}, & \\
\text { E:003 }{ }^{\prime}{ }^{\prime} 25^{\prime}, & \end{array}$} & 1 & 19.0 & 1.8 & 1.8 & Topsoil \\
\hline & 2 & 132.9 & 2.0 & 3.8 & Sandy Clay \\
\hline & 3 & 573.2 & 21.0 & 24.8 & Clayey Sand \\
\hline & 4 & 41.1 & 8.3 & 33.2 & Pegmatite+Tourmaline \\
\hline & 5 & 3166.9 & -1 & - & Fresh Basement \\
\hline \multirow{5}{*}{$\begin{array}{l}\text { VES } \\
\text { ELV:16m } \\
\text { N: } 07^{0} 58^{\prime} 20^{\prime \prime} \\
\text { E:003 } 22^{\prime} 26^{\prime \prime}\end{array}$} & 1 & 1045.2 & 0.6 & 0.6 & Topsoil \\
\hline & 2 & 36610.2 & 2.7 & 3.3 & Fresh Basement \\
\hline & 3 & 10725.2 & 3.6 & 6.9 & Fresh Basement \\
\hline & 4 & 1459.0 & 22.3 & 29.3 & Fractured Basement \\
\hline & 5 & 23349.0 & - & - & Fresh Basement \\
\hline \multirow{5}{*}{$\begin{array}{ll}\text { VES } & 13 \\
\text { ELV:164m } & \mathrm{N}: \\
07^{0} 58^{\prime} 20^{\prime}, & \\
\text { E:003 } 2^{\prime} 27^{\prime}, & \end{array}$} & 1 & 3765.9 & 0.5 & 0.5 & Topsoil \\
\hline & 2 & 1267.6 & 0.9 & 1.4 & Compacted Sandstone \\
\hline & 3 & 100000.0 & 28.3 & 29.7 & Fresh Basement \\
\hline & 4 & 100000.0 & 67.7 & 97.4 & Fresh Basement \\
\hline & 5 & 40642.9 & - & - & Fractured Basement \\
\hline \multirow{5}{*}{$\begin{array}{lr}\text { VES } & 14 \\
\text { ELV195m } & \text { N: } \\
07^{0} 58^{\prime} 20^{\prime}, & \\
\text { E:003 }^{0} 2^{\prime} 27^{\prime}, & \end{array}$} & 1 & 3407.8 & 0.6 & 0.6 & Topsoil \\
\hline & 2 & 495.4 & 0.9 & 1.5 & Clayey Sand \\
\hline & 3 & 1205.4 & 1.2 & 2.7 & Compacted Sandstone \\
\hline & 4 & 33800.6 & 18.5 & 21.2 & Fresh Basement \\
\hline & 5 & 3010.5 & & & Fresh Basement \\
\hline \multirow{5}{*}{$\begin{array}{ll}\text { VES } & 15 \\
\text { ELV:192m } & \mathrm{N}: \\
07^{0} 58^{\prime} 20^{\prime}, & \\
\text { E:003 }^{0} 2^{\prime} 27^{\prime}, & \end{array}$} & 1 & 4109.4 & 0.6 & 0.6 & Topsoil \\
\hline & 2 & 10000.0 & 4.5 & 5.1 & Fresh Basement \\
\hline & 3 & 94894.8 & 0.2 & 11.3 & Fresh Basement \\
\hline & 4 & 18586.9 & 23.6 & 35.0 & Fractured Basement \\
\hline & 5 & 100000.0 & & - & Fresh Basement \\
\hline \multirow{5}{*}{$\begin{array}{ll}\text { VES } & 16 \\
\text { ELV:192m } & \mathrm{N}: \\
07^{0} 58^{\prime} 20^{\prime}, & \\
\text { E:003 }^{0} 2^{\prime} 27^{\prime}, & \end{array}$} & 1 & 1632.9 & 0.6 & 0.6 & Topsoil \\
\hline & 2 & 724.5 & 5.6 & 6.2 & Clayey Sand \\
\hline & 3 & 106.2 & 9.6 & 15.8 & Sandy Clay \\
\hline & 4 & 648.5 & 13.2 & 29.0 & Fractured Basement \\
\hline & 5 & 6320.6 & & - & Fresh Basement \\
\hline & & & & & \\
\hline & 3 & 744.4 & 2.9 & 4.6 & Clayey Sand \\
\hline & 4 & 3722.1 & & & Fresh Basement \\
\hline
\end{tabular}


Compositional Trend in the Specks of Tantalite, Tourmaline and Beryl Hosted within Complex

\begin{tabular}{|c|c|c|c|c|c|}
\hline $\begin{array}{l}\text { VES } \\
\text { LOCATION }\end{array}$ & $\begin{array}{l}\text { LAYER } \\
\text { NO }\end{array}$ & $\begin{array}{l}\text { RESISTIVITY } \\
(\Omega \mathrm{m})\end{array}$ & $\begin{array}{l}\text { THICKNESS } \\
\text { (m) }\end{array}$ & $\begin{array}{l}\text { DEPTH } \\
(\mathrm{m})\end{array}$ & LITHOLOGY \\
\hline \multirow{4}{*}{$\begin{array}{l}\text { VES18 } \\
\text { ELV:212m N: } \\
07^{0} 58^{\prime} 21^{\prime}, \\
\text { E:003 }\end{array}$} & 1 & 999.1 & 0.2 & 0.2 & Topsoil \\
\hline & 2 & 13043.5 & 0.5 & 0.7 & Fresh Basement \\
\hline & 3 & 100000.0 & 10.3 & 11.0 & Fresh Basement \\
\hline & 4 & 12180.0 & & & Fractured Basement \\
\hline \multirow{5}{*}{$\begin{array}{ll}\text { VES } & 19 \\
\text { ELV:215m } & \mathrm{N}: \\
07^{0} 58^{\prime} 20^{\prime}, & \\
\text { E:003 } 33^{\prime} 28^{\prime},\end{array}$} & 1 & 380.0 & 0.9 & 0,9 & Topsoil \\
\hline & 2 & 393.2 & 1.8 & 2.7 & Clayey sand \\
\hline & 3 & 4550.9 & 6.2 & 8.9 & Fresh Basement \\
\hline & 4 & 420.5 & 6.8 & 15.7 & Fractured Basement \\
\hline & 5 & 2400.2 & & & Fresh Basement \\
\hline \multirow{5}{*}{$\begin{array}{ll}\text { VES } & 20 \\
\text { ELV:221m } & \mathrm{N}: \\
07^{0} 58^{\prime} 19^{\prime}, & \\
\text { E:003 } 33^{\prime} 27^{\prime},\end{array}$} & 1 & 99.2 & 0.8 & 0.8 & Topsoil \\
\hline & 2 & 56.1 & 0.8 & 1.6 & Pegmatite+Tourmaline \\
\hline & 3 & 1123.9 & 1.3 & 2.9 & Clayey Sand \\
\hline & 4 & 10900.3 & 3.0 & 5.9 & Fresh Basement \\
\hline & 5 & 1620.9 & & & Fractured Basement \\
\hline \multirow{5}{*}{$\begin{array}{ll}\text { VES } & 21 \\
\text { ELV:22m } & \mathrm{N}: \\
07^{0} 58^{\prime} 19^{\prime}, & \\
\text { E:003 } 33^{\prime} 28^{\prime},\end{array}$} & 1 & 148.2 & 0.6 & 0.6 & Topsoil \\
\hline & 2 & 148.7 & 1.1 & 1.8 & Sandy Clay \\
\hline & 3 & 248.7 & 1.1 & 2.9 & Clayey Sand \\
\hline & 4 & 5615.7 & 14.7 & 17.6 & Fresh Basement \\
\hline & 5 & 559.5 & & & Fractured Basement \\
\hline \multirow{4}{*}{$\begin{array}{ll}\text { VES } & 22 \\
\text { ELV:220m } & \mathrm{N}: \\
07^{0} 58^{\prime} 19^{\prime}, & \\
\text { E:003 } 3^{\prime} 28^{\prime},\end{array}$} & 1 & 1015.5 & 0.5 & 0.5 & Shale/Clay \\
\hline & 2 & 55.6 & 1.8 & 2.3 & $\begin{array}{ll}\text { Pegmatite } & + \\
\text { Tourmaline } & \end{array}$ \\
\hline & 3 & 14234.8 & 51.7 & 53.9 & Fresh Basement \\
\hline & 4 & 2587.7 & & & Fresh Basement \\
\hline \multirow{5}{*}{$\begin{array}{ll}\text { VES } & 23 \\
\text { ELV:221m } & \mathrm{N}: \\
07^{0} 58^{\prime} 19^{\prime}, & \\
\text { E:003 } 33^{\prime} 27^{\prime},\end{array}$} & 1 & 25.3 & 0.4 & 0.4 & Topsoil \\
\hline & 2 & 161.1 & 0.6 & 1.0 & Sandy Clay \\
\hline & 3 & 825.4 & 1.2 & 2.2 & Clayey Sand \\
\hline & 4 & 22564.3 & 17.5 & 19.7 & Fresh Basement \\
\hline & 5 & 7696.2 & & & Fresh Basement \\
\hline
\end{tabular}

TABLE 3: RESULTS OF ELECTRICAL AND THERMAL CONDUCTIVITIES MEASUREMENTS

\begin{tabular}{|l|l|l|l|}
\hline SAMPLE & $\begin{array}{l}\text { ELECTRICAL RESISTIVITY } \\
(\mathbf{\Omega m})\end{array}$ & $\begin{array}{l}\text { ELECTRICAL CONDUCTIVITY } \\
(\mathbf{S})\end{array}$ & $\begin{array}{l}\text { THERMAL } \\
\text { CONDUCTIVITY } \\
\left(\mathbf{W} / \mathbf{m}^{2} \mathbf{K}\right)\end{array}$ \\
\hline A (Beryl) & 31.0106 & 0.0322 & 201 \\
\hline B (Schist) & - & - & 250 \\
\hline $\begin{array}{l}\text { C (Black } \\
\text { Tourmaline) }\end{array}$ & 19.4106 & 0.0515 & 224 \\
\hline D (Babylon) & - & - & 229 \\
\hline E (Babylon) & - & - & 224 \\
\hline F (Tantalite) & 28.6106 & 0.0350 & 220 \\
\hline $\begin{array}{l}\text { Q(Pink } \\
\text { Tourmaline) }\end{array}$ & 19.8106 & 0.0505 & - \\
\hline & & \\
\hline
\end{tabular}

TABLE 4: SAMPLE IDENTIFICATION

\begin{tabular}{|c|c|}
\hline SAMPLES & LOCATION \\
\hline A (Beryl) & LONG.N07.97296 ${ }^{\circ}$ AND LAT.003.04096 ${ }^{\circ}$ \\
\hline B (Schist) & LONG.N07.97287 $^{\circ}$ AND LAT.003.040695 \\
\hline C (Black Tourmaline) & LONG.N07.97296 ${ }^{0}$ AND LAT.003.04096 \\
\hline D (Babylon) & LONG.N07.97276 $^{\circ}$ AND LAT.E003.04112 \\
\hline E (Babylon) & LONG.N07.97252 $2^{\circ}$ AND LAT.E003.04085 \\
\hline $\mathrm{F}$ (Tantalite) & LONG.N07.97287 ${ }^{\circ}$ AND LAT. E003.04095 \\
\hline Q1(Pink Tourmaline) & ${\text { LONG.N07. } 97269^{\circ} \text { AND LAT.E003.04109 }}^{\circ}$ \\
\hline
\end{tabular}




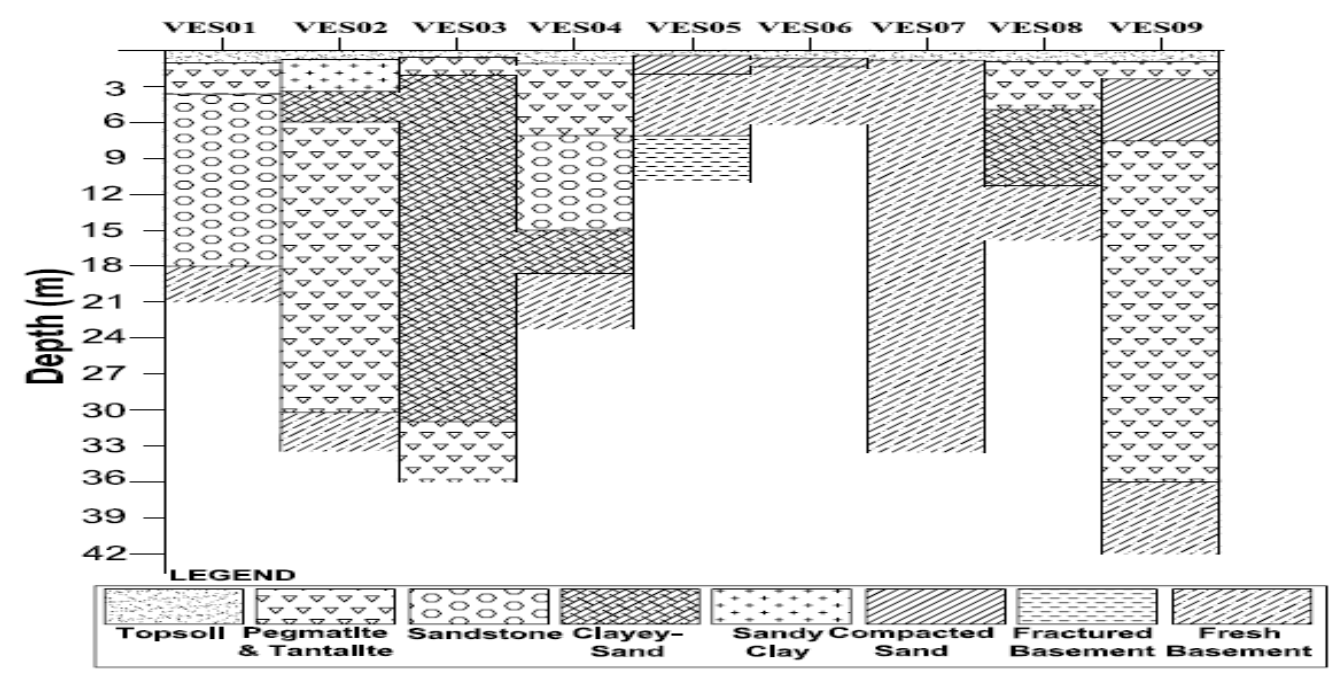

FIGURE 3: GEOELECTRIC SECTION BENEATH VES 01-09

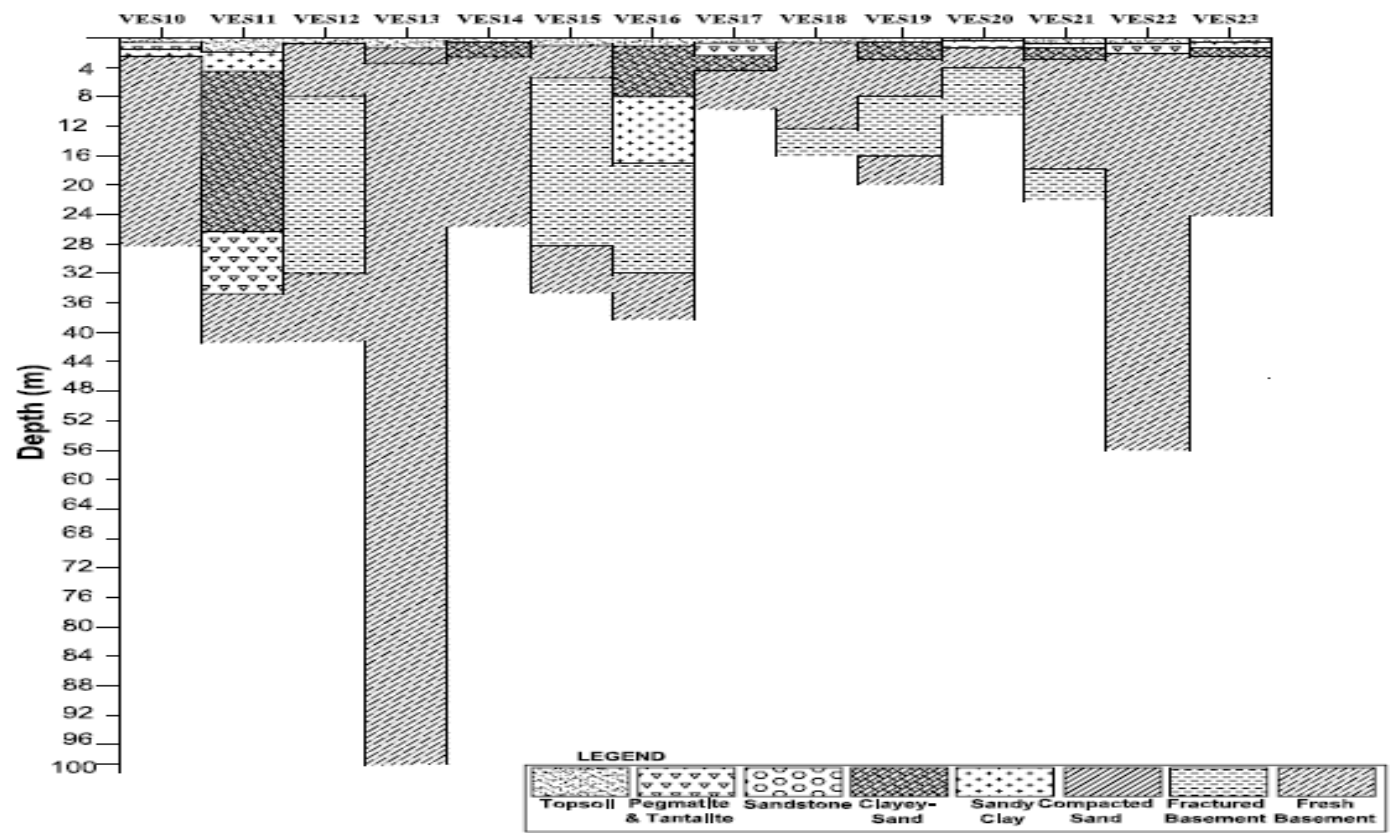

Figure 4: GEOELECTRIC SECTION BENEATH VES 10-23

\section{VES 05}

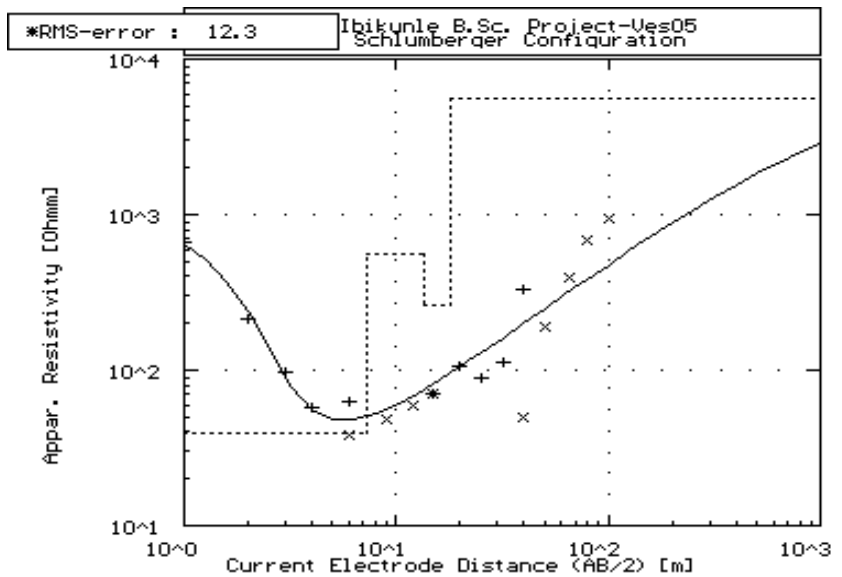

\begin{tabular}{|cccr|}
\hline No & Res & Thick & Depth \\
\hdashline 1 & 884.9 & 0.7 & 0.7 \\
2 & $39: 7$ & $6: 6$ & 7.3 \\
3 & $550: 2$ & $6: 3$ & $13: 6$ \\
4 & 261.4 & $4: 3$ & $17: 9$ \\
5 & $5613: 9$ &.-- & -9 \\
$*$ \\
RMS on smoothed data \\
\hline
\end{tabular}




\section{VES 06}
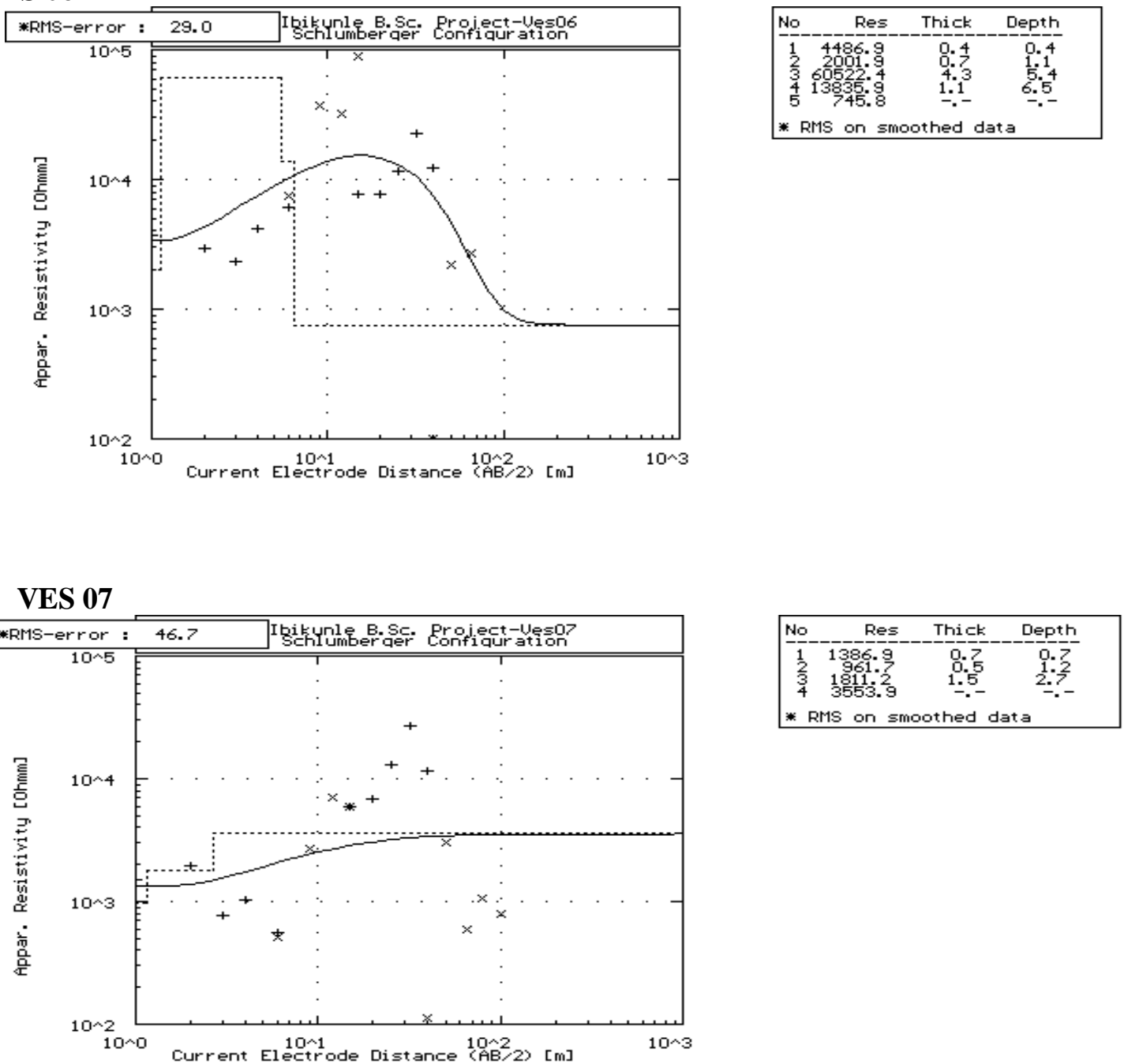

Figure 5: COMPUTER MODELING FOR SOME SELECTED SOUNDED STATIONS

\subsection{Resistivity Sounding (VES) interpretation}

\section{Discussion}

The results of the VES sounding at each of the geoelectric sections showed sounding curves with four to six geoelelctric layers as revealed by the computer iteration techniques. The resistivity values range from $2.0 \Omega \mathrm{m}-2.55 \times 10^{6} \Omega \mathrm{m}$. The pegmatites that host these solid minerals were found within the second and fourth layers (Tables 1 and 2) and also found as grains within the topsoil (VES 23). Geoelectric section beneath VES 01-09 was characterized with sparsely distributed pegmatite within VES 01, 03, 04 and 08 while VES 02 and 09 were having vast deposits of pegmatite with overburden thickness of $27.5 \mathrm{~m}$ and $24.2 \mathrm{~m}$ and resistivity values between $68.2 \Omega \mathrm{m}$ and $77.5 \Omega \mathrm{m}$ respectively. In geoelectric section beneath VES $10-23$, specks of these solid minerals were sparsely distributed in VES 10,11, 17, 20, 22 and 23 with resistivity values of 92.8, 41.1, 44.9, 56.1, 55.6 and $25.3 \Omega \mathrm{m}$ respectively. However, VES 11 has the largest deposit within this geoelectric section with thickness of $8.3 \mathrm{~m}$. For VES 23, these solid minerals were suspected within the topsoil. The solid minerals were characterized into two litho-facies changes with facies I and II located at second and fourth geoelectric layers respectively. 


\subsection{LABORATORY MEASUREMENT}

\subsubsection{Electrical Conductivity Measurement}

Electrical resistivity of rock samples (A, C, F and $\mathrm{Q}_{1}$ ) hosting tantalite, tourmaline and beryl specks were measured with electrical conductivity meter. The results revealed electrical resistivity values of tantalite, tourmaline and beryl specks to range from $19.4-31.0 \Omega \mathrm{m}$ (Table 3). This value also confirmed the classification of tourmaline and tantalite as sub-metallic material and can be found together within the same geoelectric layer.

\subsubsection{Thermal Conductivity Measurement}

Thermal conductivity measurement of samples A, B, C, D, E and F revealed the thermal conductivity of the samples to be in the range of $201-250 \mathrm{~W} / \mathrm{m}^{2} \mathrm{~K}$ (Table 3). This shows that the rate of thermal energy conduction varies from one rock sample to the other and this can serve as a basis for geothermal exploration.

\subsubsection{Geochemical Analysis TABLES 5A, B, C AND D: RESULTS OF GEOCHEMICAL ANALYSES}

\begin{tabular}{|l|l|l|l|l|l|l|}
\hline ELEMENT & $\mathrm{A}$ & $\mathrm{B}$ & $\mathrm{C}$ & $\mathrm{D}$ & $\mathrm{E}$ & $\mathrm{F}$ \\
\hline $\mathrm{Fe}: \mathrm{O}:$ & $0.87 \%$ & $7.51 \%$ & $4.69 \%$ & $4.23 \%$ & $4.33 \%$ & $4.51 \%$ \\
\hline $\mathrm{Ba}$ & 96 & 336 & 442 & 405 & 416 & 339 \\
\hline $\mathrm{Nb}$ & 0 & 12 & 10 & 10 & 18 & 12 \\
\hline $\mathrm{Ni}$ & 2 & 3 & 6 & 3 & 5 & 3 \\
\hline $\mathrm{Be}$ & 468 & 6 & 4 & 3 & 6 & 5 \\
\hline $\mathrm{B}$ & 1 & 171 & 162 & 159 & 168 & 177 \\
\hline $\mathrm{Ti}$ & 1 & 2 & 2 & 4 & 9 & 3 \\
\hline $\mathrm{Cr}$ & 4 & 4 & 1 & 4 & 6 & 3 \\
\hline
\end{tabular}

Sample $\mathrm{F}$ also analyzed for Ta and $\mathrm{Cu}$

\begin{tabular}{|l|l|l|l|}
\hline Sample F & Ta $(\mathrm{egm}) 38.9$ & Cu loom) 16.0 \\
\hline
\end{tabular}

\section{Major elements in (vit \%)}

\begin{tabular}{|l|l|l|l|l|l|l|l|l|l|}
\hline Sample & $\mathrm{SiO}:$ & $\mathrm{TiO}:$ & $\mathrm{Al} ; \mathrm{O}:$ & $\mathrm{Fe} ; \mathrm{O}:$ & $\mathrm{MnO}$ & $\mathrm{MgO}$ & $\mathrm{CaO}$ & $\mathrm{Na} ; \mathrm{O}$ & $\mathrm{K} ; \mathrm{O}$ \\
\hline $\mathrm{Q}:$ & 59.99 & 2.17 & 25.35 & 4.02 & 0.01 & 0.06 & 0.03 & 1.71 & 0.58 \\
\hline $\mathrm{Q} 2$ & 58.48 & 2.20 & 18.65 & 3.36 & 0.03 & 1.26 & 0.46 & 0.48 & 0.87 \\
\hline
\end{tabular}

Some Minor and Rare Earth Elements in pink tourmalines in PPM

\begin{tabular}{|l|l|l|l|l|l|l|l|l|l|}
\hline Sample & $\mathrm{Li}$ & $\mathrm{V}$ & $\mathrm{Ba}$ & $\mathrm{Co}$ & $\mathrm{Ni}$ & $\mathrm{Zr}$ & $\mathrm{Th}$ & $\mathrm{F}$ & $\mathrm{Ra}$ \\
\hline $\mathrm{Q}: \mathrm{A}$ & 4 & 1 & 42 & 2 & 1 & 8 & 5 & 1 & 1 \\
\hline $\mathrm{Q}: \mathrm{n}$ & 2 & 1 & 64 & 3 & 1 & 11 & 3 & 1 & 0 \\
\hline
\end{tabular}

Samples A, B, C, D, E and $\mathrm{F}$ were analyzed for $\mathrm{Fe}_{2} \mathrm{O}_{3}$ (Table 4a) with sample B having the highest proportion of 7.51\%. Samples C, D, E, and F were almost of equal proportion (4.23-4.69\%). The trace elements analyzed were $\mathrm{Ba}, \mathrm{Nb}, \mathrm{Ni}, \mathrm{Cr}, \mathrm{Ti}, \mathrm{B}$, and $\mathrm{Be}$. Sample A has the highest proportion of Be (468ppm) while others samples are between 3 and $6 \mathrm{ppm}$ in proportion (Table $5 \mathrm{a}$ ). $\mathrm{Ti}, \mathrm{Ni}, \mathrm{Cr}$ and $\mathrm{Nb}$ were present in small proportion in all samples while B and Ba were present in large proportion (Table 4a) except in sample A. The proportion of Be in sample A indicates that it is of low quality because a high quality Beryl must have over 1000ppm and other trace elements. Samples D and E are low quality tourmalines called Babylon by Artisan miners in Nigeria.

From the analysis of samples $\mathrm{Q}_{1}$ and $\mathrm{Q}_{2}$ (pink tourmalines), silica, alumina and iron were the most abundant minerals in the samples while other major elements were present in small proportion (Table5c). Ba is the most abundant trace element that was analyzed in $\mathrm{Q}_{1}$ and $\mathrm{Q}_{2}$ while other trace elements were present in small proportion (Table 4c). Zr, Th and Ra were the rare earth elements analyzed with $\mathrm{Zr}$ having the highest proportion (Table 4c). The proportion of $\mathrm{Cu}$ and $\mathrm{Ta}$ in sample $\mathrm{F}$ is 16 and 38.9ppm respectively. Sample F is 
also of low quality tantalite as revealed by $\mathrm{Ta}$ and $\mathrm{Nb}$ proportion contained. For a high quality sample of tourmalines and beryl, we have the following mineral compositions in ppm and wt.\%:

- Ni-20ppm and above

- V-20ppm and above

- $\quad \mathrm{Cr}-20 \mathrm{ppm}$ and above

- $\mathrm{FeO}-1.5 \%$ and above

\section{Conclusion}

This study has shown the compositional trends in tantalite, tourmaline and beryl as solid minerals hosted as specks within the basement rock (pegmatite and schist); using geophysical and geochemical integrated approach. The economic values of these solid minerals have been associated with the litho-facies characterization, where facies I is suspected to be black or brown tourmalines, tantalite and brown beryl and facies II is suspected to contain tourmalines and beryl of other colours. Locations of vast deposits, depth and lateral distribution have also been identified in this study area; where economic value can be derived.

[1] Kuzin, M. (1976). Field manual of minerals: pp. 148-175

[2] Jacobson, R., and Webb, J.S. (1946). The pegmatite of Central Nigeria: Goel. Surv. Nig. Bull. 17, 61 p.

[3] Ekwueme, B.N., and Matheis. G. (1995). Geochemistry and economic value of pegmatites in the Precambrian basement of southeast Nigeria: In Magmatism in relation to diverse tectonic settings (Eds. R.K. Srivastava and R. Chandra), New Delhi, Oxford and IBH Publishing Co.:375 - 392

[4] Odunaike, R.K., Ijeoma, G.C., Edigbe, R.O. and Babatope, A.H., (2009). Oil sand exploration in Ijebu-Imushin using magnetic and electrical resistivity methods.11th SAGA Biennial Technical Meeting and Exhibition Swaziland: 16-18 September 2009, pages 247-252.

[5] Badmus B.S, Iyanda J.D, Popoola I.O., (2006). Geoelectric evaluation of Mica Schists deposits in Area J $_{4}$ of Southwest, Nigeria: J. Applied Science Technology (JAST), 11(1\&2): 39-43.

[6] Badmus B.S and Ayolabi E.A., (2005). Litho-facies changes in Ewekoro limestone using Schlumberger geoelectric sounding t techniques: Journal of Applied Science and Technology (JAST), 10(1\&2): 42-52.

[7] Anamaba, S.E, Onu N.N and Iwuagwu C.J., (1993). Geophysical study of the gravel deposit in Ihiagwa, Owerri, Nigeria: Journal of Mining and Geology 29(2) 95-100.

[8] Ertl, A., Hughes, J.M., Prowatka, S., Ludwig,T., Prasad, P.S.R., Brandstatter, F., Körner, W., Schuster, R., Pertlik, F. and zarschall, H., (2006). Tetrahedrally coordinated boron in tourmalines from the Liddocoatite-elbaite series from Madagascar: Structure, chemistry and infra-red spectroscopic studies: American Mineralogist, Vol.91, 1847-1856.

[9] Abdalla, H.M., and Mohamed, F.H., (1999). Mineralogical and geochemical investigation of emerald and beryl mineralization, Pan-African belt of Egypt: genetic and exploration aspects. Jour. of Afr. Earth Sci. Vol. 28 No.3 pp581-598

[10] Rahman M.A., (1975). Review of basement geology of Southwestern Nigeria. In Geology of Nigeria (Edited by Kogbe (A) Elizabeth Nigeria.Schuiling, R.D., (1967). Tin belts on the continents around the Atlantic ocean: Econ. Geol. 62, 540-550.

[11] Wright, J.B., (1970). Controls of mineralization in the older and younger Tin fields of Nigeria: Econ.Geol. 65, 945-951.

[12] Kinnaird, J.A., 1984. Contrasting styles of Sn-Nb-Ta-Zn mineralization in Nigeria: J. Afr. Ear. Sci. Vol.2 No.2 81-90.

[13] Kuster, D., 1990. Rare metal pegmatites of Wamba, Central Nigeria- their relationship to late Pan-African granites: Mineralium Deposita 25, 25-33

[14] Mathais, G., (1979). Geochemical exploration around the pegmatitic Sn - Nb - Ta mineralization of SW Nigeria: Geol. Sci. Malaysia, Kuala Lumpur.Bull 11; 33-351.

[15] Schuiling, R.D., (1967). Tin belts on the continents around the Atlantic ocean: Econ. Geol. 62, 540-550.

[16] Cenry, P., (1989). Exploration strategy and methods for pegmatite deposits of tantalum in Moller, P. Cerny, P. and Saupe, F. (eds.), Lanthanides, Tantalum and Niobium: Soc. Geol. Appl. Min.Dep. Spec. Publ. 7. Springer Verlag 271 -299.

[17] Conklin, N.M. and Slack, J.F., (1983). Trace-element analyses of tourmaline from the Appalachian-Caledonian massive sulfide deposits: US Geological Survey Open-file Report, 83-890,5p.

[18] Ojelabi, E.A, Badmus, B.S., and Salau, A.A., (2002). Comparative Analysis of Wenner and Schlumberger Methods of Geoelectric Sounding in Subsurface Delineation and Groundwater Exploration- A case study: Journal of Geological Society of India,60, 623628 .

[19] Zohdy, A.A.R., (1965). The auxiliary point method of electrical sounding interpretation and its relationship to the Dar-Zarrouk parameters: Geophysics, 30: $644-660$

[20] Zohdy, A.A.R., Eaton, G.P. and Mabey, D.R., 1974. Application of surface geophysics to groundwater investigations: USGS TWRI book 2, chap. D1. 116p.

[21] Bhattacharya, P. K. and Patra, H.P., (1968). Direct Current Geoelectric Sounding Principles and Interpretation. Elsevier, Amsterdam, pp: 139. 